\title{
The Strangeness Magnetic Moment of the Proton in the Chiral Quark Model
}

\author{
L. Hannelius, D.O. Riska \\ Department of Physics, University of Helsinki, 00014 Finland \\ and \\ L. Ya. Glozman \\ Institute for Theoretical Physics, University of Graz, A-8019 Graz, Austria
}

\begin{abstract}
The strangeness magnetic moment of the proton is shown to be small in the chiral quark model. The dominant loop contribution is due to kaons. The $K^{*}$ loop contributions are proportional to the difference between the strange and light constituent quark masses or $m_{K^{*}}^{-2}$ and therefore small. The loop fluctuations that involve radiative transitions between $K^{*}$ mesons and kaons are small, when the cut-off scale in the loops is taken to be close to the chiral symmetry restoration scale. The net loop amplitude contribution to the strangeness magnetic moment of the proton is about -0.05 , which falls within the uncertainty range of the experimental value.
\end{abstract}




\section{Introduction}

The recent finding by the SAMPLE collaboration that the strangeness magnetic moment of the proton is small, and possibly even positive [1] $\left(G_{M}^{s}\left(Q^{2}=0.1 \mathrm{GeV}^{2}\right)=0.23 \pm 0.37\right)$ was unexpected in view of the fact that the bulk of the many theoretical predictions for this quantity are negative, and outside of the experimental uncertainty range (summaries are given e.g. in refs. [2, 3, 国). A recent lattice calculation gives $-0.36 \pm 0.20$ for this quantity [5], thus reaffirming the typical theoretical expectation, while remaining outside of the uncertainty range of the empirical value.

Under the assumption that the main strange loop contribution to the strangeness magnetic moment is the kaon loop, the expectation that $G_{M}^{s}(0)$ be negative may be explained as follows: Consider a $u$ quark with spin $s_{z}$ projection $+\frac{1}{2}$. If this fluctuates into a kaon and an $s$ quark, the probability that $s_{z}$ of the $s$ quark be $-\frac{1}{2}$ is twice that for the value $+\frac{1}{2}$ if the quarks are nonrelativistic (for ultra-relativistic quarks the $\gamma_{5}$ vertex would imply $100 \%$ helicity flip). As the charge of the $s$ quark is $-e / 3$, the $s$ quark should then contribute a positive amount to the net (positive) magnetic moment of the $u$ quark. On the other hand, following spin-flip, the $\bar{s}$ quark in the kaon will have orbital angular momentum $l_{z}=+1$, and, as its charge is $+e / 3$, it should also contribute a positive amount to the net magnetic moment of the $u$ quark. A similar argument applies for $d$ quarks. As the $u$ quarks contribute $4 / 3$ and the $d$ quarks only $-1 / 3$ to the proton magnetic moment, it follows that these strange fluctuations will give a positive contribution to the magnetic moment of the proton as usually defined. By convention $G_{M}^{s}$ is defined as the matrix element of $\bar{s} \gamma^{\mu} s$ and not of $-\frac{e}{3} \bar{s} \gamma_{\mu} s$, which matrix element is obtained by multiplication of the "conventional" magnetic moment by -3 , and thus this latter value should be negative [6]. This argument applies equally well at the hadronic level, where the corresponding strange fluctuation of the proton is into a kaon and a strange hyperon.

This general argument implies that a positive contributions to $G_{M}^{s}(0)$ can only arise from loop fluctuations that involve strange vector mesons and loops with transition couplings between different strange mesons. That is indeed borne out by calculations based on the nonrelativistic harmonic oscillator quark model [7], and by calculations of meson-hyperon loop fluctuations of baryons [6, 国. We here show that in the chiral quark model, in which the strange pseudo-scalar $(K)$ and vector $\left(K^{*}\right)$ mesons are coupled directly to the quarks, and which admits a covariant calculation, with coupling strengths determined by known hadronic couplings, these vector meson loop contributions are much smaller than those of the kaon loops. Consequently the sign of the net loop contribution is determined by the kaon loops.

To explain the reason for the smallness of the diagonal vector meson loop contribution to the strangeness magnetic moment, it is useful to separate this into the terms that arise from the $\delta_{\mu \nu}$ term in the vector meson spin-projection operator $\delta_{\mu \nu}+k_{\mu} k_{\nu} / m_{K}^{* 2}$, and the terms that arise from the second $k_{\mu} k_{\nu} / m_{k}^{* 2}$ term. The former are proportional to the quark mass difference $m_{s}-m_{u}$, and vanish in the $S U(3)_{F}$ limit $m_{s}=m_{u}=m_{d}$. This is due to a remarkable cancellation between the loop amplitudes that are associated with e.m. field coupling to the intermediate vector meson and the $s$ quark respectively. The latter terms are small because of the large meson mass squared in the denominator. As a consequence vector meson loop contributions to the strangeness magnetic moment are small. 
The present calculation should be contrasted with the calculations in refs. [4, 6], wherein the strangeness fluctuations were considered at the hadronic level and were found to give a large net negative result. The consideration of the strangeness fluctuations of the constituent quarks and their contribution to the nucleon magnetic moments here is motivated by the fact that the non-strange magnetic moments of the nucleons are explained by the constituent quark model. In contrast the hadronic model is known to fail for the anomalous magnetic moments of the nucleons, because of the large meson-nucleon coupling constants and the consequent absence of a converging loop expansion [8, 9]. The small kaon-quark coupling constant does in the present application imply loop corrections, which are sufficiently small so as not perturb the overall quark model description of the magnetic moments [10]. Moreover in the quark model approach, which automatically takes into account all baryonic intermediate states, the dominant mass dependence is due to the meson masses [7], with a consequent suppression of heavy meson loops, which is absent in the hadronic approach with large baryon masses in the energy denominators.

Baryon chiral perturbation theory does not predict the strangeness magnetic moment, as there is no other datum, which can fix the required strange counter-term in the Lagrangian density [11, 12]. A brief digression concerning the conceptual differences between the treatment of mesonic fluctuations of constituent quarks, and (directly) of baryons is in order. In the latter the mesonic loop fluctuations imply an infinite shift of the baryon mass in the chiral limit, which is balanced by phenomenologically determined counter-terms [13]. The infinite mass renormalisation of the nucleon mass may be avoided in the heavy baryon version of chiral perturbation theory, as long as no decuplet states are considered in the meson loops [14, 15]. Consideration of the mesonic degrees of freedom in such approaches yield information on corrections from the finite masses of the current quarks, but not on the origin of the nucleon mass nor on the octet-decuplet splitting in the chiral limit.

In the chiral constituent quark model [16] the role of the meson degrees of freedom is broader. On the one hand meson fluctuations contribute to the self energy of the constituent quarks, in analogy with the baryonic approach, but on the other hand they imply a strong flavour-dependent interactions between the quarks, which yield an octet-decuplet splitting already in the chiral limit [17]. The chiral quark model combines the $S U(6)$ flavour-spin structure of the nucleon wave function, which is implied in the large $N_{c}$ limit of QCD [18, 19], with the dynamical implications of spontaneous breaking of chiral symmetry. As an example both the axial current $F$ and $D$ coupling constants are predicted by the chiral quark model, while they have to be determined by experimental data in baryon effective field theory.

Some of the strange loop contributions considered here are logarithmically divergent and thus depend on the choice of a cut-off scale. The natural value for this scale is the chiral symmetry restoration scale $4 \pi f_{\pi} \sim 1.2 \mathrm{GeV}$. With such values for the cut-off a very small value for $G_{M}^{s}(0)$ obtains.

This paper falls into 5 sections. In section 2 the contributions from kaonic loops are derived, while those from diagonal $K^{*}$ loops are derived in section 3 . In section 4 the contribution to the strangeness magnetic moments of the nucleons from the strange loops, which involve a $K^{*} K \gamma$ transition is derived. Section 5 contains a concluding discussion. We use the East coast metric throughout this paper. 


\section{Kaon loop contributions}

Consider the (hidden) strangeness fluctuations $q \rightarrow K s \rightarrow q$ of $u$ and $d$ quarks that are illustrated by the Feynman diagrams in Fig. 1 (with inclusion of seagull terms as appropriate). The expressions for these contributions are similar to the corresponding expressions for the contribution of pionic fluctuation to the neutron magnetic moment [9]. For the derivation of these contributions we employ the pseudo-vector coupling for kaons to constituent quarks:

$$
\mathcal{L}_{K q s}=i \frac{f_{K q s}}{m_{K}} \bar{\psi} \gamma_{\mu} \gamma_{5} \sum_{a=4}^{7} \lambda^{a} \partial_{\mu} K^{a} \psi,
$$

and the the kaon and $s$ quark current density operators:

$$
\begin{gathered}
j_{\mu}=i e\left\{\partial_{\mu} K^{\dagger} K+\text { h.c. }\right\}, \\
j_{\mu}=-i \frac{e}{3} \bar{\psi}_{s} \gamma_{\mu} \psi_{s} .
\end{gathered}
$$

Here the underlying assumption is that the strange quark in the loop may be approximately described by a free fermion propagator, with a constant constituent mass in the relevant low momentum region. With the usual (unusual) convention of assigning the $s$ quark a "strangeness charge" of 1 , and the kaon a "strangeness charge" of -1 , the kaon current (2.2a) should be multiplied by -1 and the $s$ quark current (2.2b) by -3 .

The pseudo-vector kaon-quark coupling constant $f_{K q s}$ may be related to the kaon decay constant $f_{K}$ as

$$
f_{K q s}=\frac{g_{A}^{q}}{2} \frac{m_{K}}{f_{K}} .
$$

Here $g_{A}^{q}$ is the axial coupling constant for the constituent quark, for which we use the value $g_{A}^{q}=0.87$ [20, 21]. With $f_{K}=113 \mathrm{MeV}$ we then obtain $f_{K q s}=1.9$.

The pseudo-vector coupling (2.1) implies a contact current term, which generates two seagull diagrams in addition to the loop diagrams in Fig. 1. The contribution from these seagull diagrams cancels the corresponding contact terms that obtain from the diagrams in Fig. 1, once the Dirac equation for the external quark lines is used. The remaining term corresponds to the loop diagrams obtained if the the kaons couple to quarks by pseudo-scalar coupling:

$$
\mathcal{L}_{K q s}=i g_{K q s} \psi \gamma_{5} \sum_{a=4}^{7} \lambda^{a} K^{a} \psi,
$$

where

$$
g_{K q s}=\frac{m_{q}+m_{s}}{m_{K}} f_{K q s} .
$$

Here $m_{q}$ and $m_{s}$ represent the constituent masses of the $u, d$ and $s$ quarks respectively. For the latter we employ the values $m_{q}=340 \mathrm{MeV}$ and $m_{s}=460 \mathrm{MeV}$ [16]. This then yields the value $g_{\text {Kqs }}^{2} / 4 \pi=0.75$ for the effective coupling strength of the kaon loop diagrams. The smallness of this value indicates a converging loop expansion.

While the kaon loop contributions to the strangeness magnetic moment is finite even in the absence of regularisation, we impose a cut-off at the chiral restoration scale $4 \pi f_{\pi} \sim 1.2$ 
GeV. The cut-off may be imposed by multiplying the kaon propagator $v\left(k^{2}\right)=1 /\left(k^{2}+m_{K}^{2}\right)$ in the loop diagram, in which the coupling is to the current of the $s$ quark (Fig. 1a), by a squared monopole form factor:

$$
v\left(k^{2}\right) \rightarrow \frac{1}{m_{K}^{2}+k^{2}}\left(\frac{\Lambda^{2}-m_{K}^{2}}{\Lambda^{2}+k^{2}}\right)^{2} .
$$

Maintenance of the current conservation condition on the sum of the two kaon loop contributions in Fig. 1 then requires that the two kaon propagators in the loop amplitude, in which the coupling is to the kaon current (Fig. 1b), be modified in the following way [22]:

$$
\frac{1}{m_{K}^{2}+k_{1}^{2}} \frac{1}{m_{K}^{2}+k_{2}^{2}} \rightarrow \frac{v\left(k_{2}^{2}\right)-v\left(k_{1}^{2}\right)}{k_{1}^{2}-k_{2}^{2}} .
$$

These kaonic loops then give the same contribution to the anomalous strangeness magnetic moment of the $u$ and $d$ quarks. By the standard quark model result

$$
\mu(p)=\frac{1}{3}[4 \mu(u)-\mu(d)]
$$

the strangeness magnetic moment contribution to the proton from these loop diagrams is then $\mu_{s}(p)=\mu_{s}(u)$. The explicit expressions for the contributions from the two loop diagrams in Figs. $1 \mathrm{a}$ and $\mathrm{b}$ are then

$$
\begin{aligned}
& G_{M}^{s}(0)\{a, K\}=-\frac{g_{K q s}^{2}}{4 \pi^{2}} \frac{m_{p}}{m_{q}} \int_{0}^{1} d x(1-x)^{2} \frac{m_{q}\left(m_{s}-m_{q} x\right)}{H\left(m_{K}^{2}\right)}, \\
& G_{M}^{s}(0)\{b, K\}=-\frac{g_{K q s}^{2}}{4 \pi^{2}} \frac{m_{p}}{m_{q}} \int_{0}^{1} d x x(1-x) \frac{m_{q}\left(m_{s}-m_{q} x\right)}{H\left(m_{K}^{2}\right)},
\end{aligned}
$$

Here the function $H\left(m_{K}^{2}\right)$ is defined as

$$
H\left(m_{K}^{2}\right)=m_{s}^{2}(1-x)-m_{q}^{2} x(1-x)+m_{K}^{2} x .
$$

It follows from (2.9) that the net kaon loop contribution to the strangeness magnetic moments of the proton is

$$
G_{M}^{s}(0)\{K\}=G_{M}^{s}(0)\{a, K\}+G_{M}^{s}(0)\{b, K\} .
$$

The expressions (2.9) and (2.10) do not take into account the cut-off form factor (2.7),(2.8). The effect of the form factor is however readily taken into account by substitution of the factor $1 / H\left(m_{K}^{2}\right)$ in $(2.10)$ by the replacement

$$
\frac{1}{H\left(m_{K}^{2}\right)} \rightarrow K\left(m_{K}^{2}\right) \equiv \frac{1}{H\left(m_{K}^{2}\right)}-\frac{1}{H\left(\Lambda^{2}\right)}-x \frac{\Lambda^{2}-m_{K}^{2}}{H\left(\Lambda^{2}\right)^{2}}
$$

The values of the expression (2.12) are shown in Fig. 2 as functions of the cut-off parameter $\Lambda$. For $\Lambda$ below $1.2 \mathrm{GeV}$ the kaon loop contribution is small and negative (as expected), and does not exceed $-0.064 \mu_{N}$ in magnitude. Note the limit

$$
\lim _{\Lambda \rightarrow \infty} \lim _{m_{K} \rightarrow 0} \lim _{m_{s} \rightarrow m_{q}} G_{M}^{s}(0)\{K\}=-\frac{g_{K q s}^{2}}{4 \pi^{2}} \frac{m_{p}}{m_{q}} .
$$


If $g_{K q s}$ is replaced by the pseudo-scalar pion-nucleon coupling constant, and $m_{q}$ is replaced by the nucleon mass, this limit agrees with the well known value for the contribution of the $\pi^{-} p$ loop fluctuation to the anomalous magnetic moment of the neutron [9].

\section{3. $K^{*}$ loop contributions}

To calculate the loop fluctuations that involve $K^{*}$ mesons illustrated by the Feynman diagrams in Fig. 1, when the meson lines represent strange vector mesons, we employ the Lagrangian that describes the coupling of constituent quarks to $K^{*}$ mesons.

$$
\mathcal{L}_{K^{*} q s}=i \frac{g_{K^{*} q s}}{m_{K}^{*}} \bar{\psi}\left(\gamma_{\mu}+i \frac{\kappa_{K^{*} q s}}{2 \bar{m}} \sigma_{\mu \nu} \partial_{\nu}\right) \sum_{a=4}^{7} \lambda^{a} K_{\mu}^{* a} \psi .
$$

Here $\bar{m}$ represents the mean constituent mass $\bar{m}=\left(m_{q}+m_{s}\right) / 2$. The vector $\left(g_{K^{*} q s}\right)$ and ratio of tensor to vector coupling constants $\left(\kappa_{K^{*} q s}\right)$ may be determined from the corresponding $K^{*}$ - baryon octet coupling constants by the quark model relations [23]:

$$
\begin{gathered}
g_{K^{*} q s}=g_{K^{*} \bar{B} B}, \\
g_{K^{*} q s}\left(1+\kappa_{K^{*} q s}\right)=\frac{3}{5} \frac{\bar{m}}{\bar{M}} g_{K^{*} \bar{B} B}\left(1+\kappa_{K^{*} \bar{B} B}\right) .
\end{gathered}
$$

Here $\bar{M}$ represents the average of the nucleon and $\Lambda$ hyperon masses $(\bar{M}=1027 \mathrm{MeV})$.

The nucleon-hyperon coupling constants may be determined indirectly by fitting potential models to nucleon-hyperon scattering data. A recent such determination gives $g_{K^{*} \bar{B} B}=2.97$ and $\kappa_{K^{*} \bar{B} B}=4.22$, although with a considerable uncertainty margin [24. From (3.2) we then have $g_{K^{*} q s}^{2} / 4 \pi \simeq 0.7$ and $\kappa_{K^{*} q s} \simeq=0.21$. In view of the smallness of the tensor coupling for the quarks we shall neglect it here. While the vector coupling constant is large a substantial cancellation makes the contribution from the $K^{*}$ loops very small, as shown below.

The current density operator for the $K^{* \pm}$ mesons has the form

$$
j_{\mu}= \pm i e\left\{K_{\nu}^{* \dagger} \partial_{\mu} K_{\nu}^{*}-K_{\nu}^{* \dagger} \partial_{\nu} K_{\mu}^{*}\right\}+\text { h.c. }
$$

The vector meson propagator is $-i /\left(m_{K}^{* 2}+k^{2}\right)\left(\delta_{\mu \nu}+k_{\mu} k_{\nu} / m_{K}^{* 2}\right)$. With the current density operators $(2.2 \mathrm{~b}),(3.3)$ and the coupling (3.1) the combination of the amplitudes that correspond to the two vector meson loop diagrams in Fig. 1 yield a conserved current.

The two vector meson loop diagrams in give the same contributions to the anomalous strangeness magnetic moments of the $u$ and $d$ quarks, and by (2.9) these contributions equal that for the corresponding strangeness magnetic moment of the proton.

The quark current loop amplitude (Fig. 1a) gives the following contribution to $G_{M}^{s}(0)$ :

$$
\begin{gathered}
G_{M}^{s}(0)\left\{a, K^{*}\right\}=2 \frac{g_{K^{*} q s}^{2}}{4 \pi^{2}} \frac{m_{p}}{m_{q}} \int_{0}^{1} d x\left\{m_{q} x(1-x)\left[2 m_{s}-m_{q}(1+x)\right] K\left(m_{K}^{* 2}\right)\right. \\
-\frac{1}{2} \frac{m_{q}^{2}}{m_{K}^{* 2}}(1-x)\left[(1-x)^{2}\left(m_{s}-m_{q}\right)\left(m_{s}+m_{q} x\right) K\left(m_{K}^{* 2}\right)\right.
\end{gathered}
$$




$$
\left.\left.-2\left(1-\frac{m_{s}}{m_{q}}\right)\left(1-\frac{3}{2} x\right)\left(\ln \frac{H\left(\Lambda^{2}\right)}{H\left(m_{K}^{* 2}\right)}-x \frac{\Lambda^{2}-m_{K}^{* 2}}{H\left(\Lambda^{2}\right)}\right)\right]\right\} .
$$

The function $K\left(m_{K}^{* 2}\right)$ is defined in (2.13). A check of this expression is possible by comparison to the anomalous magnetic moment of the electron. Consider the limit

$$
\lim _{\Lambda \rightarrow \infty} \lim _{m_{K^{*} \rightarrow 0}} \lim _{m_{s} \rightarrow m_{q}} G_{M}^{s}(0)\left\{a, K^{*}\right\}=\frac{g_{K^{*} q s}^{2}}{4 \pi^{2}} \frac{m_{p}}{m_{q}} .
$$

For comparison to the anomalous magnetic moment of the electron divide out the flavour factor 2 and change the sign to make allowance for the negative electron charge. The result then reduces to the standard value $-\alpha / 2 \pi$ for the anomalous magnetic moment of the electron if the quark and proton masses are replaced by the electron mass, and $\alpha$ is substituted for $g_{K^{*} q s}^{2} / 4 \pi$.

The contribution from the $K^{*}$ current loop amplitude (Fig. 1b) to $G_{M}^{s}(0)$ is

$$
\begin{aligned}
G_{M}^{s}(0)\left\{b, K^{*}\right\}=- & \frac{g_{K^{*} q s}^{2}}{4 \pi} \frac{m_{p}}{m_{q}} \int_{0}^{1} d x x\left\{m_{q}\left[m_{s}(2-3 x)-m_{q} x(1-2 x)\right] K\left(m_{K}^{* 2}\right)\right. \\
& -\frac{m_{q}^{2}}{m_{K}^{* 2}}\left[m_{q}(1-x)^{2}\left(m_{s}-m_{q} x^{2}\right) K\left(m_{K}^{* 2}\right)\right. \\
-2\left(\frac{m_{s}}{m_{q}}-\right. & \left.\left.\left.2(1-x)^{2}-x\right)\left(\ln \frac{H\left(\Lambda^{2}\right)}{H\left(m_{K}^{* 2}\right)}-x \frac{\Lambda^{2}-m_{K}^{* 2}}{H\left(\Lambda^{2}\right)}\right)\right]\right\} .
\end{aligned}
$$

The net $K^{*}$ loop contribution to the strangeness magnetic moment of the proton is then [cf. (2.12)]

$$
G_{M}^{s}(0)\left\{K^{*}\right\}=G_{M}^{s}(0)\left\{a, K^{*}\right\}+G_{M}^{s}(0)\left\{b, K^{*}\right\} .
$$

This contribution to the strangeness magnetic moment of the proton is also shown in Fig. 2 . The vector meson loop contributions is much smaller in magnitude than the kaon loop contribution. It changes sign at $\Lambda \sim 1.2 \mathrm{GeV}$. The smallness of the vector meson loop contribution may also be understood from the fact that the sum of the main terms in (3.4) and (3.5) (the terms without the factor $1 / m_{K^{*}}^{2}$ ) is proportional to the (small) quark mass difference $m_{s}-m_{q}$, and vanishes in the equal mass limit for the constituent quarks. The other terms are small, as they are proportional to $1 / m_{K}^{* 2}$. There is no a priori reason to expect the vector meson loop contributions to have any specific sign, as both the spin non-flip charge as well as the spin-flip current couplings contribute to the net result. In the ultra-relativistic limit, only the helicity conserving charge coupling survives, and with a consequent positive loop contribution.

The cancellation of the main vector meson loop contributions to the strangeness magnetic moment in the equal quark mass limit is remarkable, and also applies for loop contributions considered at the hadronic level in the case of pure Dirac couplings. The large $K^{*}$ loop contributions found in ref. [4] can therefore only be understood as consequences of large tensor couplings. 


\section{Strangeness Loops with Transition Couplings}

We finally consider the (hidden) strangeness fluctuations of light $u$ and $d$ quarks, which involve the radiative transition coupling $K^{*} \rightarrow K \gamma$ (Fig. 3). The key vertex in this loop diagram is the $K^{*} \rightarrow K \gamma$ vertex, which is described by the transverse current matrix element:

$$
\left\langle K^{a}\left(k^{\prime}\right)\left|J_{\mu}\right| K_{\sigma}^{* b}(k)\right\rangle=-i \frac{g_{K^{*} K \gamma}}{m_{K}^{*}} \epsilon_{\mu \lambda \nu \sigma} k_{\lambda} k_{\nu}^{\prime} \delta^{a b} .
$$

The coupling constant $g_{K^{*} K \gamma}$ is determined by the empirical decay widths for radiative decay of the $K^{*}$ as

$$
\Gamma\left(K^{*} \rightarrow K \gamma\right)=\alpha \frac{g_{K^{*} K \gamma}^{2}}{24} m_{K}^{*}\left[1-\left(\frac{m_{K}}{m_{K}^{*}}\right)^{2}\right]^{3},
$$

where $\alpha$ is the fine structure constant and $m_{K}$ is the mass of the kaon. From the empirical decay widths $\Gamma\left(K^{*+} \rightarrow K^{+} \gamma\right)=50 \mathrm{keV}$ and $\Gamma\left(K^{* 0} \rightarrow K^{0} \gamma\right)=116 \mathrm{keV}$ 25 we obtain $g_{K^{*+} K^{+\gamma}}=0.75$ and $g_{K^{* 0} K^{0} \gamma}=1.14$.

As the decay widths do not determine the signs of these coupling constants, we fix those by invoking $S U(3)$ flavour symmetry to link the vertices (2.1) to the corresponding vertex for radiative decay of the $\rho$ meson. The sign of the $g_{\rho \pi \gamma}$ coupling is ultimately determined by the triangle anomaly, or in the Skyrme model by the Wess-Zumino term through the topological baryon current and determines the sign of the $\rho \pi \gamma$ exchange current contribution to the iso-scalar exchange current of nuclei [26, 27]. With this justification, the signs above obtain.

As the $K^{*} K s$ loop diagrams are logarithmically divergent, a cut-off has to be invoked in their evaluation. Such is introduced by insertion of monopole form factors of the form $\left(\Lambda^{2}-m_{K}^{2}\right) /\left(\Lambda^{2}+k_{1}^{2}\right)$ and $\left(\Lambda^{* 2}-m_{K}^{* 2}\right) /\left(\Lambda^{2 *}+k_{2}^{2}\right)$ at the $K$ and $K^{*}$ vertices respectively. The cut-off mass $\Lambda^{*}$ is taken equal to that of $\Lambda$ in the numerical calculations here.

Given the current matrix element (4.1) and the Lagrangian densities (2.5), (3.1a) the contributions to the anomalous magnetic moment of the $u$ and $d$ quarks from the $K^{*} K \gamma$ loop diagrams (Fig. 3) take the following form (when expressed in terms of nuclear magnetons and after assigning the kaon line a "strangeness charge" of -1$)$ :

$$
\begin{gathered}
G_{M}^{s}(0)_{u, d}=-\frac{g_{K q s} g_{K^{*} q s} g_{K^{*} K \gamma}}{2 \pi^{2}} \frac{m_{p}}{m_{K}^{*}} \int_{0}^{1} d x x \\
\times \int_{0}^{1} d y\left\{m_{q}(1-x)\left(m_{s}-m_{q} x\right)\left(\frac{1}{G_{1}}-\frac{1}{G_{2}}-\frac{1}{G_{3}}+\frac{1}{G_{4}}\right)-\ln \left(\frac{G_{2} G_{3}}{G_{1} G_{4}}\right)\right\} .
\end{gathered}
$$

Here $m_{p}$ is the proton mass and $m_{s}$ the constituent mass of the $s$ quark, which we shall take to have the value $460 \mathrm{MeV}$ [16]. The quantities $G_{j}$ above are defined as

$$
G_{1}=G\left(m_{K}, m_{K^{*}}\right), G_{2}=G\left(\Lambda, m_{K^{*}}\right), G_{3}=G\left(m_{K}, \Lambda^{*}\right), G_{4}=G\left(\Lambda, \Lambda^{*}\right),
$$

where the function $G$ is defined as

$$
G\left(m, m^{\prime}\right)=m_{s}^{2}(1-x)-m_{q}^{2} x(1-x)+m^{2} x(1-y)+m^{\prime 2} x y .
$$

In the case of the $u$ quark the coupling $g_{K^{*} K \gamma}$ constant is that for the $K^{+}, K^{*+}$ mesons and in the case of the $d$ quark it is that for the $K^{0}, K^{* 0}$ mesons. 
The contribution from these loops to the strangeness magnetic moment of the proton is obtained as

$$
G_{M}^{s}(0)\left\{K, K^{*}\right\}=\frac{4}{3} G_{M}^{s}(0)_{u}\left\{K, K^{*}\right\}-\frac{1}{3} G_{M}^{s}(0)_{d}\left\{K, K^{*}\right\}
$$

and thus will have a value close to the corresponding constituent quark values.

In Fig. 2 we show the contribution from the $K^{*} K s$ loop to the strangeness magnetic moment of the $u$ and $d$ quarks as functions of the cut-off parameter $\Lambda$. For $\Lambda$ values in the range $1-1.5 \mathrm{GeV}$ the contributions are positive, but small (Table 1 ).

These values are somewhat smaller than the corresponding value obtained in ref. [7] with the harmonic oscillator quark model. The difference is mainly due to the fact that the latter is non-covariant, and consequently overestimates the radiative widths of the vector mesons. With the usual static fermion currents of the constituent quark model the coupling constant $g_{K^{*} K \gamma}$ should be 2, which is about twice too large. Even so it best to view the results in Fig. 2 as representing lower estimates from of the strange fluctuations, which involve radiative transition couplings of the form $K^{*} \rightarrow K \gamma$, as the higher lying vector mesons $K^{*}(1410)$ should also contribute. As the radiative widths of these vector excited strange vector mesons are not known, their contribution cannot be calculated without an explicit quark model at this time. If their radiative widths should be as large as the empirical radiative widths of the strange tensor mesons $K_{2}^{*}(1430)$ the curves in Fig. 2 would lie about $30 \%$ higher than those shown.

\section{Discussion}

The main result obtained here is that the strangeness magnetic moment of the proton is small. When the cut-off parameter has values close to the chiral symmetry restoration scale, the net value for it is about $-0.05 \mathrm{n} . \mathrm{m}$. The smallness of the (dominant) kaon fluctuations is a consequence of the small effective K-quark coupling constant. The diagonal strange vector meson loop fluctuations are small because of a cancellation between the two loop diagrams in Fig. 1, when the meson lines represent $K^{*}$ mesons. In the $S U(3)_{F}$ limit the vector meson

loop contributions cancel exactly, with exception for the terms that arise from the $k_{\mu} k_{\nu} / m_{K^{*}}^{2}$ terms in the vector meson propagator, which terms are small because of the large mass of the $K^{*}$. The strangeness fluctuations that involve heavier strange mesons will be similarly suppressed by the larger meson masses. A systematic treatment of such will have to await the empirical determination of the radiative widths for $K \gamma$ decay of all strange mesons with masses below $1.5 \mathrm{GeV}$ (At this time only the radiative widths of the tensor mesons $K_{2}^{*}(1430)$ in this region are known). In principle the quark model calculation of ref. [7] takes into account all such effects, although it is schematic in being nonrelativistic and in its description of the $K$ mesons as pure quark-antiquark states rather than as the collective states implied by their role as Goldstone bosons of the spontaneously broken approximate chiral symmetry. The nonrelativistic description of the $K^{*} K \gamma$ vertex leads to an overestimate of the associated loop fluctuation.

The present approach recommends itself over the hadronic loop calculations in refs. ઔ, 6] by the fact that it appears to account reasonably well also for the non-strange baryon magnetic moments (once the appropriate exchange current contributions are included [28]) 
and by the fact that the chiral quark model, with the meson quark couplings used above, also describes the baryon spectrum itself fairly well [29]. While in baryon effective field theory the anomalous parts of the baryon magnetic moments are exclusively due to loop contributions, the bulk of these are already implied by the $S U(6)_{F S}$ structure of the wave functions in the quark model, leaving room for only very small loop contributions [10]. That this should be so is natural as the loop contribution to the anomalous magnetic moments at the quark level should be suppressed by $\left(m_{q} / \Lambda_{\chi}\right)^{2} \sim 0.1$, where $\Lambda_{\chi}=4 \pi f_{\pi}$ is the chiral restoration scale.

The small magnitude of the net predicted strangeness magnetic moment $\sim-0.05$ is fairly insensitive to the value of the cut-off parameter $\Lambda$. It falls within the uncertainty range of the empirical value, and is also commensurate with recent more phenomenological results [31. The result suggests that the strangeness radius of the proton should be small, if it - in analogy with the result the neutron charge radius [10, 30] - it is dominated by the second (Foldy) term in the expression

$$
\left\langle r_{s}^{2}\right\rangle=-6 \lim _{q^{2} \rightarrow 0} \frac{d}{d q^{2}} F_{1 s}\left(q^{2}\right)+\frac{3}{2} \frac{F_{2 s}(0)}{m_{n}^{2}}
$$

Since $F_{2 s}(0)=G_{M}^{s}(0)$ is small, only a remarkably strong momentum dependence in the strange Dirac form factor $F_{1 s}$ would lead to a substantial value for the strangeness radius.

The only predictions for $G_{M}^{s}$, which are large and positive $(0.37,0.41)$ have been obtained in the chiral bag model [32, 33] and a linear quark-soliton model [34], both of which treat strangeness as a collective zero mode, with large phenomenological flavour symmetry breaking terms. There is no obvious way to connect these results to those obtained by more conventional phenomenological models. The bound state version of the topological soliton model [35], in which the hyperons are described as bound states of kaons and topological solitons, would give the same (negative) sign for the strangeness magnetic moment as the conventional hadronic loop calculation.

\section{Acknowledgements}

This work was supported in part by the Academy of Finland under contract 43982. L. H. thanks the Finnish Society of Sciences and Letters for support. D. O. R. thanks Professor R. D. McKeown for instructive discussions.

\section{References}

[1] B. Mueller et al., Phys. Rev. Lett. 78 (1997) 3824

[2] M. J. Musolf et al., Phys. Rept. 239 (1994) 1

[3] E. J. Beise et al., Proc. SPIN96 symposium, eprint nucl-ex/9610011

[4] L. L. Barz et al., Nucl. Phys. A640 (1998) 259 
[5] S. J. Dong, K. F. Liu and A. G. Williams, eprint hep-ph/9712483

[6] M. J. Musolf and M. Burkhardt, Z. Phys. C61 (1994) 433

[7] P. Geiger and N. Isgur, Phys. Rev. 55 (1997) 299

[8] A. Houriet, Helv. Phys. Acta 18 (1945) 473

[9] H. A. Bethe and F. de Hoffman, Mesons and Fields, Vol. II Row, Peterson, Evanston (1955)

[10] L. Ya. Glozman and D. O. Riska, Physics Letters B459 (1999) 49

[11] M. J. Ramsay-Musolf and H. Ito, Phys. Rev. C55 (1997) 3066

[12] T. R. Hemmert, U.-G. Meissner and S. Steininger, Phys. Lett. B437 (1998) 184

[13] J. Gasser, M. E. Sainio and A. Svarc, Nucl. Phys. B307 (1988) 779

[14] E. Jenkins and A. Manohar, in Effective field theories of the standard model, U.-G. Meissner ed., World Scientific, Singapore (1992) 113

[15] E. Jenkins and A. Manohar, Phys. Lett. B255 (1991) 558

[16] L. Ya. Glozman and D. O. Riska, Phys. Rept. 268 (1996) 263

[17] L. Ya. Glozman, Physics Letters B459 (1999) 589

[18] R. F. Dashen, E. Jenkins and A. Manohar, Phys. Rev. D49 (1994) 4713

[19] R. F. Dashen, E. Jenkins and A. Manohar, Phys. Rev. D51 (1995) 3697

[20] S. Weinberg, Phys. Rev. lett. 65 (1990) 1181

[21] D. A. Dicus et al., Phys. Lett. B284 (1992) 384

[22] F. Gross and D. O. Riska, Phys. Rev. C36 (1987) 1228

[23] D. O. Riska and G. E. Brown, hep-ph/9902319

[24] T. A. Rijken and V. G. J. Stoks, Phys. Rev. C59 (1999) 21

[25] C. Caso et al., The European Physical Journal C3 (1998) 1

[26] E. M. Nyman and D. O. Riska, Phys. Rev. Lett. 57 (1986) 3007, Nucl. Phys. A468 (1987) 473

[27] M. Wakamatsu and W. Weise, Nucl. Phys. A477 (1988) 559

[28] K. Dannbom et al., Nucl. Phys. A616 (1997) 555

[29] L. Ya. Glozman et al., Phys. Rev. D58 (1998) 094030 
[30] Yu. A. Aleksandrov, Phys. Part. and Nucl. 30 (1999) 29

[31] U. -G. Meissner, V. Mull and J. Speth, Phys. Lett.B408 (1997) 381

[32] S.-T. Hong, B.-Y. Park, Nucl. Phys. A561 (1993) 525

[33] S.T. Hong, B.-Y. Park and D. P. Min, Phys. Lett. B414 (1997) 229

[34] H.-C. Kim et al., Phys. Rev. D58 (1998) 114027

[35] C. G. Callan, K. Hornbostel and I. Klebanov, Phys. Lett. B202 (1988) 269 
Table 1

Contributions to the strangeness magnetic moment of the proton (in nuclear magnetons) from the loops with $K^{*} \rightarrow K \gamma$ transition vertices (Fig. 1) and with diagonal $K K \gamma$ vertices (Fig. 3) as a function of the cut-off parameter $\Lambda$ (in MeV). The chiral symmetry restoration scale corresponds to $\Lambda_{\chi} \sim 1.2 \mathrm{GeV}$

\begin{tabular}{|l|l|l|l|l|}
\hline$\Lambda$ & $K K \gamma$ & $K^{*} K^{*}$ & $K^{*} K \gamma$ & Sum \\
\hline 700 & -0.014 & 0.003 & -0.005 & -0.015 \\
800 & -0.024 & 0.0005 & -0.003 & -0.027 \\
900 & -0.035 & 0.000002 & 0.0004 & -0.035 \\
1000 & -0.045 & 0.0002 & 0.005 & -0.040 \\
1100 & -0.055 & 0.0003 & 0.011 & -0.043 \\
1200 & -0.064 & 0.00008 & 0.018 & -0.046 \\
1300 & -0.072 & -0.0005 & 0.025 & -0.048 \\
1400 & -0.080 & -0.001 & 0.032 & -0.049 \\
1500 & -0.087 & -0.003 & 0.040 & -0.049 \\
\hline
\end{tabular}




\section{Figure Captions}

Fig. 1 The $K s$ and $K^{*} s$ loop fluctuations with diagonal couplings, which contribute to the strangeness magnetic moments of the $u$ and $s$ quarks and to $G_{M}^{s}(0)$ of the proton. In the case of the $K^{*}$ loops the meson lines represents $K^{*}$ mesons.

Fig. 2 The contribution to $G_{M}^{s}(0)$ of the proton: $K$ loops ("Kaon"), $K^{*}$ loops (" $K^{*}$ "), $K^{*} K$ loops (" $\left.K^{*}-K^{\prime}\right)$ The net result is given by the curve "Total".

Fig. 3 The $K^{*} K s$ fluctuations of $u$ and $d$ quarks, which involve the radiative transition coupling $K^{*} \rightarrow K \gamma$. 


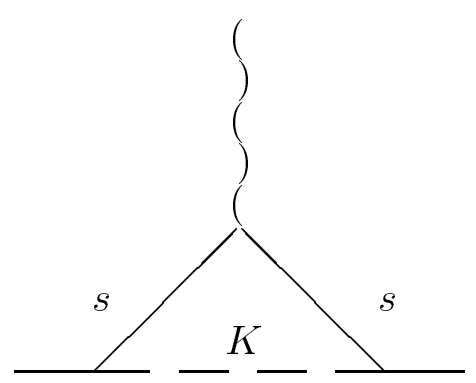

(a)

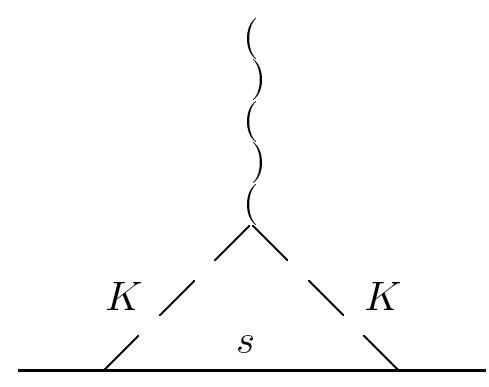

(b)

Fig. 1 
Fig. 2

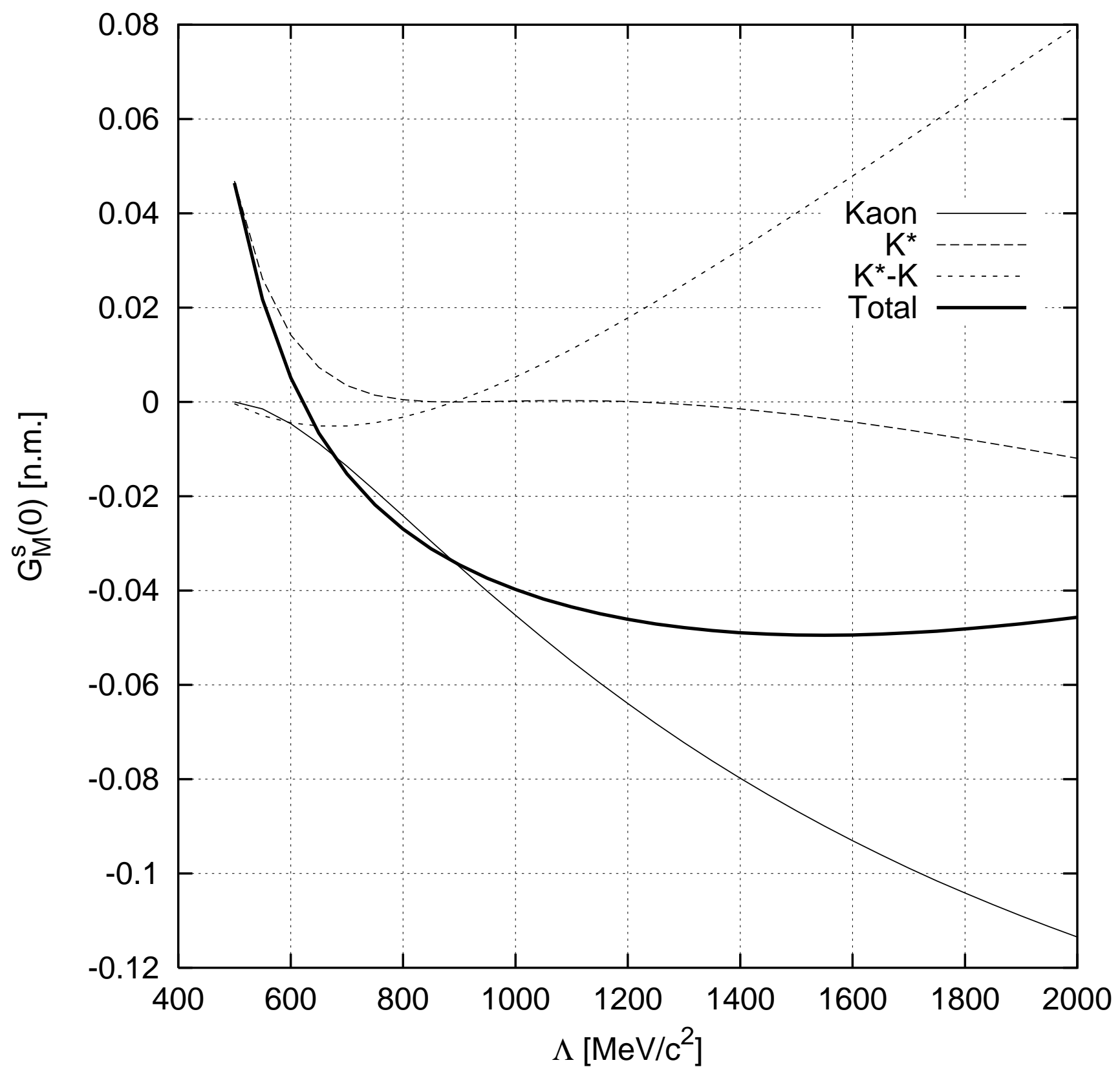




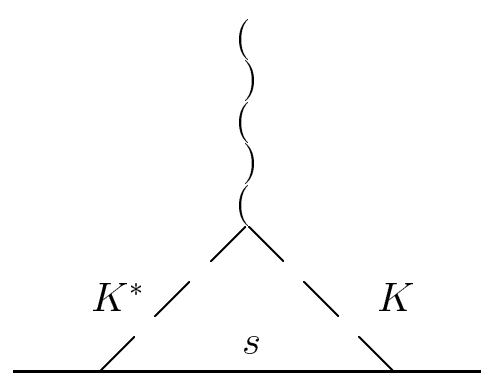

(a)

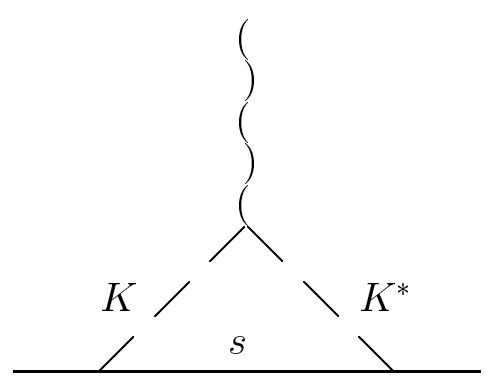

(b)

Fig. 3 